\title{
Effect of lentil sprouts on glycemic control in overweight and obese patients with type 2 diabetes
}

\author{
Zahra Aslani $^{1}$, Beitollahe Alipour ${ }^{2}$, Zahra Bahadoran ${ }^{1}$, Farzane Bagherzadeh $^{2}$, Parvin Mirmiran $^{1}$ \\ ${ }^{1}$ Nutrition and Endocrine Research Center, and Obesity Research Center, Research Institute for Endocrine Sciences, Shahid Beheshti \\ University of Medical Sciences, Tehran, Iran \\ ${ }^{2}$ Department of Nutrition in Society, Nutrition Faculty, Tabriz University of Medical Sciences, Tabriz, Iran
}

Email address:

mirmiran@endocrine.ac.ir (P. Mirmiran)

\section{To cite this article:}

Zahra Aslani, Beitollahe Alipour, Zahra Bahadoran, Farzane Bagherzadeh, Parvin Mirmiran. Effect of Lentil Sprouts on Glycemic Control in Overweight and Obese Patients with Type 2 Diabetes. International Journal of Nutrition and Food Sciences. Special Issue: Functional Foods and Nutraceuticals for Management of Type 2 Diabetes. Vol. 4, No. 2-1, 2015, pp. 10-14.

doi: $10.11648 /$ j.ijnfs.s.2015040201.13

\begin{abstract}
Health benefits of Lentil sprout (LS) have not yet been well documented. In this study, effects of LS consumption on glycemic parameters and anthropometric measurements in overweight and obese patients with type 2 diabetes were investigated. Thirty-nine type 2 diabetic patients were randomly divided into two groups; LS group and controls. Patients in LS group received $60 \mathrm{~g}$ lentil sprouts daily, and the patients in control group continued regular diet. Anthropometric measurements, the levels of fasting blood glucose (FBS), hemoglobin A1C (HbA1c), serum insulin, homeostasis model assessment of insulin resistance (HOMA-IR) and quantitative insulin sensitivity check index (QUICKI) were assessed at baseline and after 8-weeks. After 8 weeks, serum levels of HbA1c and HOMA-IR were lower in the LS group compared to control group $(P<0.05)$. The QUICKI also was higher in the LS group compared to controls $(0.34$ vs. $0.32, P<0.05)$. The results suggested that LS consumption could have favorable effects on glycemic control in overweight and obese patients with type 2 diabetes.
\end{abstract}

Keywords: Lentil Sprouts, Type 2 Diabetes, Glycemic Control, Anthropometric Measurements

\section{Introduction}

Diabetes mellitus is a metabolic disorder characterized by absolute or relative insulin deficiency, hyperglycemia, and disorder in carbohydrate and lipid metabolism [1]. Glycosilated hemoglobin (HbA1c) is an important index in blood glucose controlling in the last 2-3 months [2-4]. Also $\mathrm{HbAlc}$ is an independent risk factor for $\mathrm{CVD}_{\mathrm{s}}$ diseases in people with or without diabetes [5,6]. Due to side effects of drugs which are used to treat diabetes, using complementary therapies and modification of the dietary pattern are good ways to improve the condition of disease. Various studies have shown that legumes consumption has many effects in health improvement, control and protection against metabolic diseases such as type 2 diabetes and $\mathrm{CVD}_{\mathrm{s}}$ [7]. The fiber content of lentil seed is 3.7 gr per 100 gr; lentil seed also has low glycemic index (21/2). After germination of the lentil seeds, the amount of fiber and protein increased [8]. As the effects of lentil sprouts (LS) on glycemic parameters and anthropometric measurements in diabetic patients with overweight and obesity have not yet been determined, according to the importance of legumes and cereal sprouts in Iranian traditional medicine, we therefore conducted this trial to investigate the effect of LS on fasting blood glucose, HbA1c, insulin concentration, quantitative insulin sensitivity check index (QUICKI), homeostasis model assessment of insulin resistance (HOMA-IR) and anthropometric measurements in overweight and obese patients with type 2 diabetes.

\section{Methods}

Methods are presented as blow:

\subsection{LS Preparation}

The lentil seeds were placed in water for 30 hours. The seeds were placed on a wet cotton tissue. After 24 hours, lentil sprouts were packed and sent to patients. In these stages temperature of environment was $25-30^{\circ} \mathrm{C}$, but after the preparation they were kept in a refrigerator. 


\subsection{Study Population}

Patients [male and female] with type 2 Diabetes, who were referred to the Iran Diabetes society and endocrine clinic of Taleghani medical center, were evaluated for inclusion criteria. This trial was done between September and November 2013. Ethics approval was obtained from ethical committee of Tabriz University of medical science. The trial has been registered in the Iranian Registry of Clinical trial at http://www.irct.ir with the following identification IRCT201305251640N9. The patients aged 30-65years, having body mass index $25-40 \mathrm{~kg} / \mathrm{m}^{2}$. Patients were defined as type 2 diabetes patients who have been suffered for at least one year. Subjects with renal, hepatic disorders, gestation or lactation and patients who have used insulin injection, contraceptive pills, glucocorticoid drugs or LS and multivitamin and supplements with antioxidant during 3 months before the beginning of the study were excluded. None of the participants used alcohol or tobacco products. Forty-eight subjects (31 men and 17 women) were enrolled and divided into two groups randomly. Subjects in the control group (16 men and 7 women) received only standard drug therapy; however, the LS group (15 men and 10 women) consumed 60 -grams of LS per day during 8 weeks with standard drug therapy. Patients in the LS group consumed LS alone or with salad. Subjects provided with written informed consent. For monitoring assess nutrient intakes, three-day dietary recalls including two weekdays and one weekend day were collected at baseline and again after eight weeks from participants. The method of consumption was written for the participants. They were asked to maintain their habitual life style, physical activity and dietary pattern during the eight weeks of study. Patients were excluded from the analysis if they consumed $<85 \%$ of the packets or changed their medication or reported severe side effects.

\subsection{Anthropometric and Biochemical Measurements}

Anthropometric measurements including weight, height and waist circumference were measured at baseline and after 8 weeks. Weight was measured with a scale (Seca, Hamburg, Germany) while the subjects were minimally clothed without shoes. Height was measured with $0.5-\mathrm{cm}$ accuracy, in a standing position without shoes, by using seca tape meter. Body mass index (BMI) was calculated as follow: weight/ square of height $\left(\mathrm{Kg} / \mathrm{m}^{2}\right)$. Waist circumference of participants was measured using a tape meter in standing position [9]. Hip circumference was measured at the maximum bump hips. Waist to hip ratio (WHR) and waist-to-height ratio (WHtR) were calculated as follow: waist circumference/hip circumference and waist circumference/height. Blood pressure of the subjects was measured by standardized mercury sphygmomanometer after 15-min rest in sitting position and on the right arm. For each patient two measurements of blood pressure were taken. Venous blood samples were drawn after an overnight fast in baseline and at the end of experimental periods (Days 0 and 56) and were centrifuged at $4^{\circ} \mathrm{C}$ and $500 \mathrm{~g}$ for $10 \mathrm{~min}$ to separate plasma. Fasting blood glucose (FBS) was measured by enzymatic colorimetric method using a glucose oxidation kit (pars Azmun Company, Tehran, Iran).
Insulin serum concentration was evaluated by using ELISA kits (mercodia Company, Uppsala, Sweden). HbA1c assessment kits were provided from Millan (Italy) company. The quantitative insulin sensitivity check index (QUICKI) was calculated by the inverse of the sum of the logarithms of the fasting insulin and fasting glucose: $1 /$ (log (fasting insulin $\mu \mathrm{U} / \mathrm{mL}$ ) $+\log$ (fasting blood glucose $\mathrm{mg} / \mathrm{dL}$ ) [10]. The homeostatic model assessment (HOMA) is a method used to quantify insulin resistance and beta-cell function. It was evaluated by this formula: fasting blood glucose $(\mathrm{mml} / \mathrm{L}) \times$ insulin concentration $(\mu \mathrm{U} / \mathrm{mL}) / 22.5$ [11]. It was considered HOMA- IR $>5$ as insulin resistance and HOMA- IR $<3$ as none insulin resistance [12].

\subsection{Statistical Analysis}

According to statistical methods for triglyceride variable [13], with confidence interval (CI) $95 \%$ and $80 \%$ power, the sample size with regard to possible loss of the samples was calculated among 20 patients in each group. The experimental data was analyzed by statistical package for the social sciences (SPSS software, version16; SPSS Inc., Chicago, IL, USA). The normality of the distribution of variables was determined by the Kolmogorov- Smirnov test. Analysis of covariance was used to identify any differences between two groups after intervention, adjusting for baseline measurements and covariates. The changes in anthropometric measurements, lipid profile and ox-LDL parameters of the participants between the beginning and end of the trial were compared by paired sample t-tests. The percent change for each variable was also calculated by formula [(8- weeks values- baseline values)/baseline values $\times 100]$. Also, $p$ - value $<0.05$ was considered significant.

\section{Results}

Table 1. Baseline characteristics and biochemical values of diabetic patients in control and lentil sprout groups. ${ }^{a}$

\begin{tabular}{lll}
\hline & Control & Lentil sprouts \\
\hline Age $($ year) & $54 \pm 7.4$ & $52 \pm 7.6$ \\
Women/men $(n)$ & $7 / 13$ & $5 / 14$ \\
Duration of diabetes & $7.4 \pm 6.6$ & $11.7 \pm 19.2$ \\
Glucose lowering drugs $(n)$ & 13 & 19 \\
Lipid lowering drugs $(n)$ & 2 & 9 \\
Blood pressure lowering drugs $(n)$ & 3 & 7 \\
Weight $(\mathrm{kg})$ & $78.5 \pm 10.5$ & $78.3 \pm 9.8$ \\
BMI $\left(\mathrm{kg} / \mathrm{m}^{2}\right)$ & $28.2 \pm 2.6$ & $29.4 \pm 3.6$ \\
WC $(\mathrm{cm})$ & $98.9 \pm 8$ & $97.9 \pm 7.7$ \\
WHR & $0.8 \pm 0.03$ & $0.8 \pm 0.04$ \\
WHtR & $0.6 \pm 0.04$ & $0.6 \pm 0.05$ \\
Insulin $(\mathrm{m} U / \mathrm{L})$ & $8.9 \pm 4.4$ & $8.1 \pm 5.3$ \\
HbA1c & $7.4 \pm 1.2$ & $8.3 \pm 1.3$ \\
HOMA-IR & $3.4 \pm 1.7$ & $3.2 \pm 2.1$ \\
QUICKI & $0.32 \pm 0.02$ & $0.34 \pm 0.04$ \\
FBS $(\mathrm{mg} / \mathrm{dl})$ & $162 \pm 55$ & $159 \pm 44$ \\
\hline
\end{tabular}

FBS, fasting blood glucose; BMI, body mass index; WC, waist circumference; WHR, weight- to- hip ratio; WHtR, weight- to- height ratio; HOMA-IR, (Hemeostasis model assessment)- Insulin resistance; QUICKI, (Quantitative insulin sensitivity check index) 
Forty-eight subjects were enrolled and divided into two groups randomly, but only 39 were available for data analysis, because some of them could not complete the entire protocol or come for secondary evaluation. These 39 patients were included from 12 women and 27 men (Fig.1). No significant differences between the two groups were observed for mean age, duration of diabetes, weight, BMI, WC, WHR, WHtR, blood lowering pressure drugs; nor were there any significant differences between the pretreatment serum values of $\mathrm{HbA1c}$, insulin concentration, FBS, HOMA and QUICKI between the two groups at baseline (Table 1). Subjects' demographics are shown in Table 1. There were significant differences in lipid lowering and Anti-diabetic drugs between two groups, therefore these variables were adjusted in the analysis. Eightweek biochemical values of the participants in two groups and treatment effect of LS on FBS, HbAlc, insulin concentration, QUICKI, HOMA, BMI, WC, WHR and WHtR are presented in table 2. Figure. 2 presents the mean percent changes of variables in both groups after 8 weeks intervention. In the LS group the level of serum $\mathrm{HbA} 1 \mathrm{c}$ significantly decreased compared with baseline but in the control group no significant change was seen. HOMA-IR and FBS tended to decrease in the LS group compared with baseline. The level of QUICKI increased compared with baseline in the LS group. Patients in the intervention group did not report any side- effects for LS consumption.

${ }^{\mathrm{a}}$ All values are mean $\pm \mathrm{SE}$.

Table 2. Anthropometric and glycemic parameters of the patients after 8 weeks in control and lentil sprouts groups. ${ }^{a}$

\begin{tabular}{llll}
\hline & Control & Lentil sprouts & $\begin{array}{l}\text { P for treatment } \\
\text { effect }\end{array}$ \\
\hline WC $(\mathrm{cm})$ & $98.6 \pm 0.3$ & $98.3 \pm 0.31$ & 0.5 \\
WHR & $0.85 \pm 0.002$ & $0.85 \pm 0.002$ & 0.49 \\
WHtR & $0.59 \pm 0.002$ & $0.59 \pm 0.002$ & 0.51 \\
BMI $\left(\mathrm{kg} / \mathrm{m}^{2}\right)$ & $29.1 \pm 0.1$ & $28.9 \pm 0.1$ & 0.50 \\
Insullin $(\mathrm{mU} / \mathrm{L})$ & $10.2 \pm 0.8$ & $8.1 \pm 0.9$ & 0.07 \\
HOMA-IR & $3.8 \pm .32$ & $2.7 \pm 0.35$ & 0.02 \\
QUICKI & $0.32 \pm 0.01$ & $0.34 \pm 0.01$ & 0.01 \\
HbA1c $\%$ & $8.3 \pm 0.3$ & $7.5 \pm 0.3$ & 0.05 \\
FBS $(\mathrm{mg} / \mathrm{dl})$ & $160 \pm 7.1$ & $149 \pm 7.5$ & 0.22 \\
\hline
\end{tabular}

WC, waist circumference; WHR, waist to hip ratio; WHtR, waist-to- height ratio; BMI,body mass index, HOMA-IR; Hemeostasis model assessmentInsullin Resistance; QUICKI, quantitative insulin sensitivity check index; HbA1c, Glycated haemoglobin; FBS, fasting blood glucose

${ }^{\mathrm{a}}$ All values are mean $\pm \mathrm{SE}$.

${ }^{\mathrm{b}}$ calculated by using general linear model with 8 -week values as dependent variables, baseline values as covariates and treatment group as a fix factor. ${ }^{*}$ Significantly different from baseline values (student paired $t$ - test) $\mathrm{P}<0.05$.

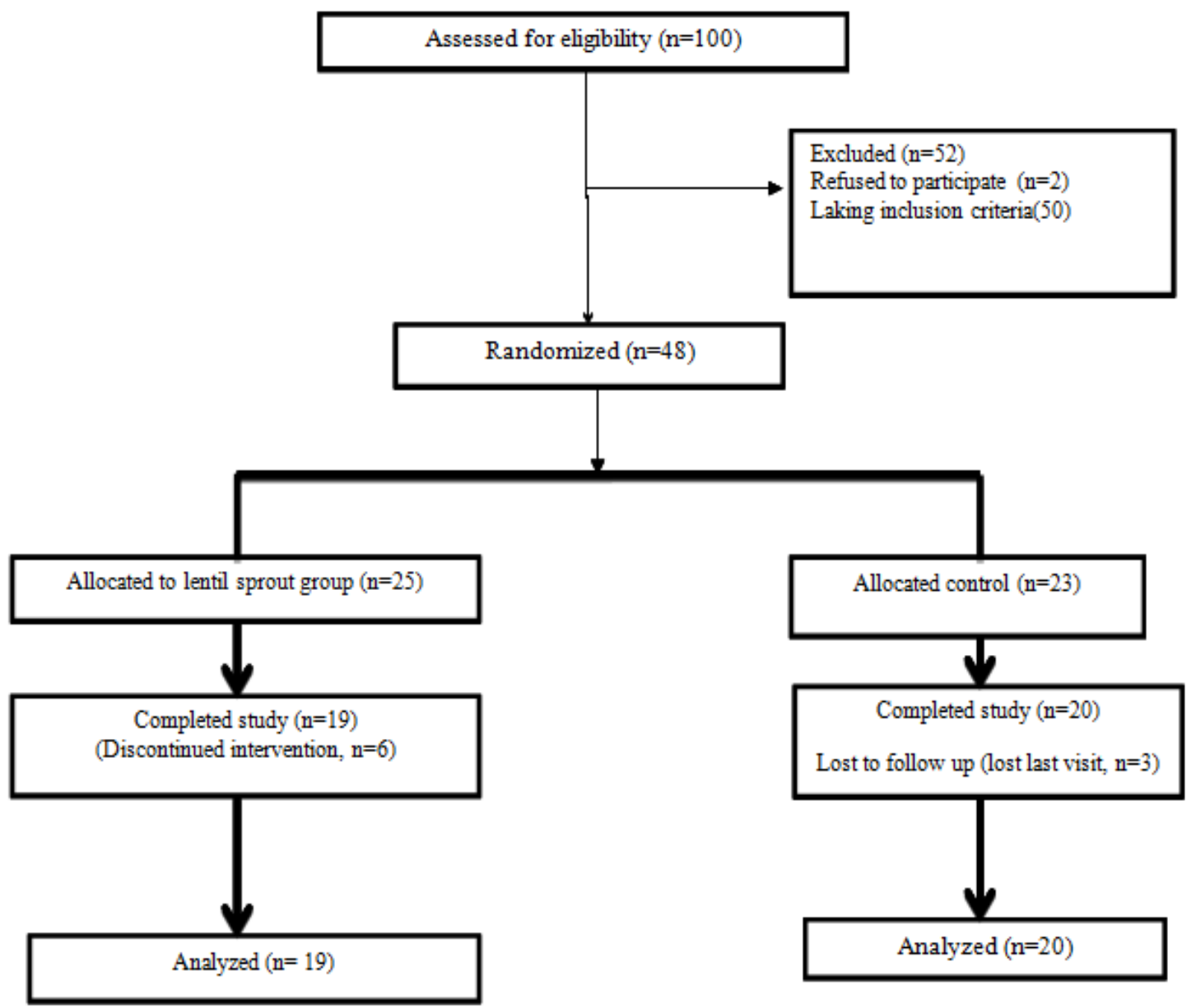

Figure 1. Flow chart of the study participants. 


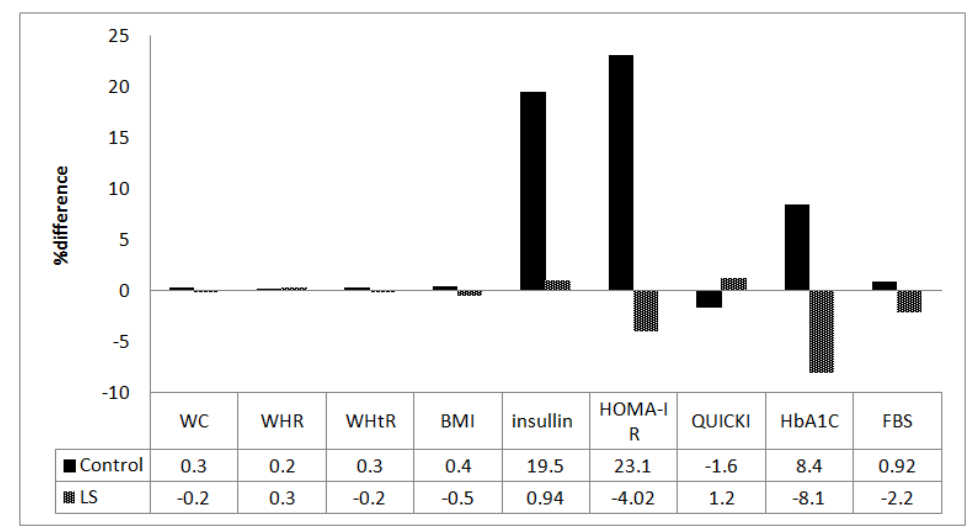

Figure 2. Mean differences of variables compared with baseline values in two groups (*significant difference within the groups using paired t-test, $p<0.05$ ). FBS, fasting blood glucose; HbAIC, Glycated hemoglobin, Insullin; HOMA-IR, homeostasis model assessment- Insullin resistance; QUICKI, quantitative insulin sensitivity check index ; WC, waist circumference; WHR, waist to hip ratio; WHtR, waist- to- height- ratio ; BMI, body mass index .

\section{Discussion}

In the current study the effects of LS consumption on overweight and obese patients with type 2 diabetes were investigated. LS consumption in type 2 diabetic patients for 8 weeks had favorable effects on and glycemic control in overweight and obese patients with type 2 diabetes. Lentil sprouts consumption had not any adverse effects in the LS group. Increase in $\mathrm{BMI}>25$ resulted metabolic disorders especially between patients with metabolic syndrome or insulin resistance [14]. Vascuer at el showed in one metaanalysis study that WC, WHR and BMI were same in diabetes predicting [15]. Spietch at el reported that patients consumed low glycemic index (LGI) food, their BMI was lower than patients who consumed low fat diet [16]. Ludwig at el released that participants consumed LGI food had more satiety than people consumed high glycemic index (HGI) food, also intake LGI food decreased appetite and desire to receive food [17]. Some studies showed that HGI food consumption resulted weight increase in people $[18,19]$. In one study after LGI food consumption, without limitation in carbohydrate and low fat diet weight reduction was significant after 6 months [20]. In this study anthropometric indexes (WC, WHtR and BMI) tend to decrease in the LS group compared with baseline, but in the control group no reduction effects were seen (Fig.2). In one cohort study results reported that between patients experienced $\mathrm{CVD}_{\mathrm{s}}$ more than other people, $\mathrm{HbAlc}$ was higher [21]. Jenkins at el found that HbAlc and FBS were significantly lower between patients with type 2 diabetes when they consumed LGI foods [22]. Results of some studies on noncommunicable diseases showed that glycemic load (GL) and glycemic index (GI) of diet are independent risk factors for $\mathrm{CVD}_{\mathrm{s}}$ and type 2 diabetes [23]. HbA1c was lower in diabetic people when they consumed LGI and high fiber food compared with diabetic people when they consumed HGI and low fiber food [24]. HbAlc was lower in patients with type 2 diabetes than patients with type 2 diabetes when their diet has higher GI and GL [25]. 12-13 unit reduction in GI caused $39 \%-50 \%$ decrease in $\mathrm{HbAlc}$ [26]. In this study $\mathrm{HbAlc}$ reduced in the LS group. 8\% decrease in HbAlc in our study, in the LS group may be clinically valuable. Insulin resistance related to metabolic syndrome and type 2 diabetes [27]. In our study HOMA-IR reduced after 8 weeks LS consumption. Diet which is rich in fruits and vegetables due to plant fibers, carotenoids, anti-oxidants and phytosterols can prevent and control non-communicable diseases such as diabetes [28]. Herbal antioxidants had insulin-like effects and increased glucose absorption in peripheral tissues [29]. Lentils had a higher oxygen radical absorbing capacity (ORCA) value than most of the common fruits and vegetables such as apples, plums, blackberries, cherries, figs, peaches, pears, oranges, garlic, cabbage and almonds [30]. Increase in QUICKI level may be resulted from these components. The present study has a few limitations because the sample size was small and the study duration was 8 weeks.

\section{Conclusion}

Consumption of LS as supplementary treatment in type 2 diabetes could have favorable effects on $\mathrm{HbAlc}$, FBS, QUICKI, and HOMA-IR. Further study with larger sample size and longer duration is required to confirm favorable effects lentil sprouts.

\section{Acknowledgements}

This study was supported by a grant from faculty of Nutrition (Tabriz University of Medical sciences).

\section{Declaration of Interest}

The authors thank all volunteers for taking part in this study. The authors report no conflicts of interest.

\section{References}

[1] Akbar S, Bellary S, Griffiths HR. Dietary antioxidant interventions in type 2 diabetes patients: a meta-analysis. Br. J. Of Diabetes and Vascular Medicine. 2011;11(2):62-8. 
[2] Larijani B, Abolhasani F, Mohajeri-Tehrani MR, Tabtabaie O. Prevalence of diabetes mellitus in Iran in 2000. IJDM. 2005;4(3):75-83.

[3] Burtis CA, Ashwood ER, Bruns DE. Tietz textbook of clinical chemistry and molecular diagnostics: Elsevier Health Sciences; 2012.

[4] Procopiou M. [HbA1c: review and recent developments]. Rev Med Suisse. 2006;2(68):1473-79.

[5] Windler E. What is the consequence of an abnormal lipid profile in patients with type 2 diabetes or the metabolic syndrome? Atherosclerosis. 2005;6(3):11-4.

[6] Esteghamati A, Abbasi M, Nakhjavani M, Yousefizadeh A, Basa AP, Afshar H. Prevalence of diabetes and other cardiovascular risk factors in an Iranian population with acute coronary syndrome. Cardiovasc. Diabetol. 2006;5(1):15.

[7] Englyst KN, Vinoy S, Englyst HN, Lang V. Glycaemic index of cereal products explained by their content of rapidly and slowly available glucose. BJN. 2003;89(03):329-39.

[8] Asgari E, Rahmani k, Taslimi A. Physicochemical properties of conventional andcomplementary foods made from wheat or lentil sprouts. J. Food Sci.. 2008;1(3):33-44.

[9] Williamson DF, Kahn HS, Worthman CM, Burnette JC, Russell CM. Precision of recumbent anthropometry. AM J HUM BIOL. 1993;5(2):159-67.

[10] Vanhala P, Vanhala M, Kumpusalo E, Keinanen-Kiukaanniemi $\mathrm{S}$. The quantitative insulin sensitivity check index QUICKI predicts the onset of type 2 diabetes better than fasting plasma insulin in obese subjects: a 5-year follow-up study. J Clin Endocrinol Metab. 2002;87(12):5834-7.

[11] Matthews D, Hosker J, Rudenski A, Naylor B, Treacher D, Turner R. Homeostasis model assessment: insulin resistance and $\beta$-cell function from fasting plasma glucose and insulin concentrations in man. Diabetologia. 1985;28(7):412-9.

[12] Miyazaki Y, Pipek R, Mandarino L, DeFronzo RA. Tumor necrosis factor $\alpha$ and insulin resistance in obese type 2 diabetic patients. Int. J. Obes. 2003;27(1):88-94.

[13] Afaghi A, Ziaee A, Afaghi M. Effect of low-glycemic load diet on changes in cardiovascular risk factors in poorly controlled diabetic patients. Indian $\mathrm{J}$ Endocrinol Metab. 2012;16(6):991.

[14] Meigs JB, Wilson PW, Fox CS, Vasan RS, Nathan DM, Sullivan LM, et al. Body mass index, metabolic syndrome, and risk of type 2 diabetes or cardiovascular disease. J Clin Endocrinol Metab. 2006;91(8):2906-12.

[15] Vazquez G, Duval S, Jacobs DR, Silventoinen K. Comparison of body mass index, waist circumference, and waist/hip ratio in predicting incident diabetes: a meta-analysis. Epidemiol. Rev. 2007;29(1):115-28.

[16] Spieth LE, Harnish JD, Lenders CM, Raezer LB, Pereira MA, Hangen SJ, et al. A low-glycemic index diet in the treatment of pediatric obesity. Arch Pediatr Adolesc Med. 2000;154(9):947-51.
[17] Ludwig DS. Dietary glycemic index and obesity. J. Nutr;130(2):280S-3S.

[18] Liu S, Willett WC, Stampfer MJ, Hu FB, Franz M, Sampson L, et al. A prospective study of dietary glycemic load, carbohydrate intake, and risk of coronary heart disease in US women. Am. J. Clin. Nutr. 2000;71(6):1455-61.

[19] Anderson GH, Woodend D. Effect of Glycemic Carbohydrates on Short-term Satiety and Food Intake. Nutr rev. 2003;61(s5):S17-S26.

[20] Ebbeling CB, Leidig MM, Sinclair KB, Seger-Shippee LG, Feldman HA, Ludwig DS. Effects of an ad libitum lowglycemic load diet on cardiovascular disease risk factors in obese young adults. The American journal of clinical nutrition. 2005;81(5):976-82.

[21] Ikeda F, Doi Y, Ninomiya T, Hirakawa Y, Mukai N, Hata J, et al. Haemoglobin A1c even within non-diabetic level is a predictor of cardiovascular disease in a general Japanese population: the Hisayama study. Cardiovasc Diabetol. 2013;12:164.

[22] Jenkins D, Wolever T, Buckley G, Lam K, Giudici S, Kalmusky J, et al. Low-glycemic-index starchy foods in the diabetic diet. AJCN. 1988;48(2):248-54.

[23] Salmeron J, Manson JE, Stampfer MJ, Colditz GA, Wing AL, Willett WC. Dietary fiber, glycemic load, and risk of noninsulin-dependent diabetes mellitus in women. Jama. 1997;277(6):472-7.

[24] Jiang J, Qiu H, Zhao G, Zhou Y, Zhang Z, Zhang H, et al. Dietary Fiber Intake Is Associated with HbAlc Level among Prevalent Patients with Type 2 Diabetes in Pudong New Area of Shanghai, China. PloS one. 2012;7(10):e46552.

[25] Esposito K, Maiorino MI, Di Palo C, Giugliano D. Dietary glycemic index and glycemic load are associated with metabolic control in type 2 diabetes: The CAPRI experience. Metab Syndr Relat 2010;8(3):255-61.

[26] Willett W, Manson J, Liu S. Glycemic index and glycemic load in the prevention and treatment of type 2 diabetes mellitus . Arg Bras Endocrinol Metabol. 2009;53(5):560-71.

[27] Matsuda M, DeFronzo RA. Insulin sensitivity indices obtained from oral glucose tolerance testing: comparison with the euglycemic insulin clamp. Diabetes care. 1999;22(9):1462-70.

[28] Urooj A, Puttaraj S. Glycaemic responses to cereal-based Indian food preparations in patients with non-insulindependent diabetes mellitus and normal subjects. BJN. 2000;83(05):483-8.

[29] Madani H, Ahmady Mahmoodabady N, Vahdati A. Effects of hydroalchoholic extract of Anethum graveolens (Dill) on plasma glucose an lipid levels in diabetes induced rats. IJDM 2005;5(2):109-16.

[30] Low PA, Nickander KK, Tritschler HJ. The roles of oxidative stress and antioxidant treatment in experimental diabetic neuropathy. Diabetes. 1997;46(Supplement 2):S38-S42. 